Muséologies

Les cahiers d'études supérieures

muséologies

\title{
Les musées québécois sont-ils à l'avant-garde en matière de gestion de collections. Survol comparatif des politiques d'acquisition des musées québécois, américains et anglais
}

\section{Sophie Deschamps}

Volume 1, numéro 1, octobre 2006

URI : https://id.erudit.org/iderudit/1033659ar

DOI : https://doi.org/10.7202/1033659ar

Aller au sommaire du numéro

Éditeur(s)

Association Québécoise de Promotion des Recherches Étudiantes en Muséologie (AQPREM)

ISSN

1718-5181 (imprimé)

1929-7815 (numérique)

Découvrir la revue

Citer cet article

Deschamps, S. (2006). Les musées québécois sont-ils à l'avant-garde en matière de gestion de collections. Survol comparatif des politiques d'acquisition des musées québécois, américains et anglais. Muséologies, 1(1), 80-97.

https://doi.org/10.7202/1033659ar 


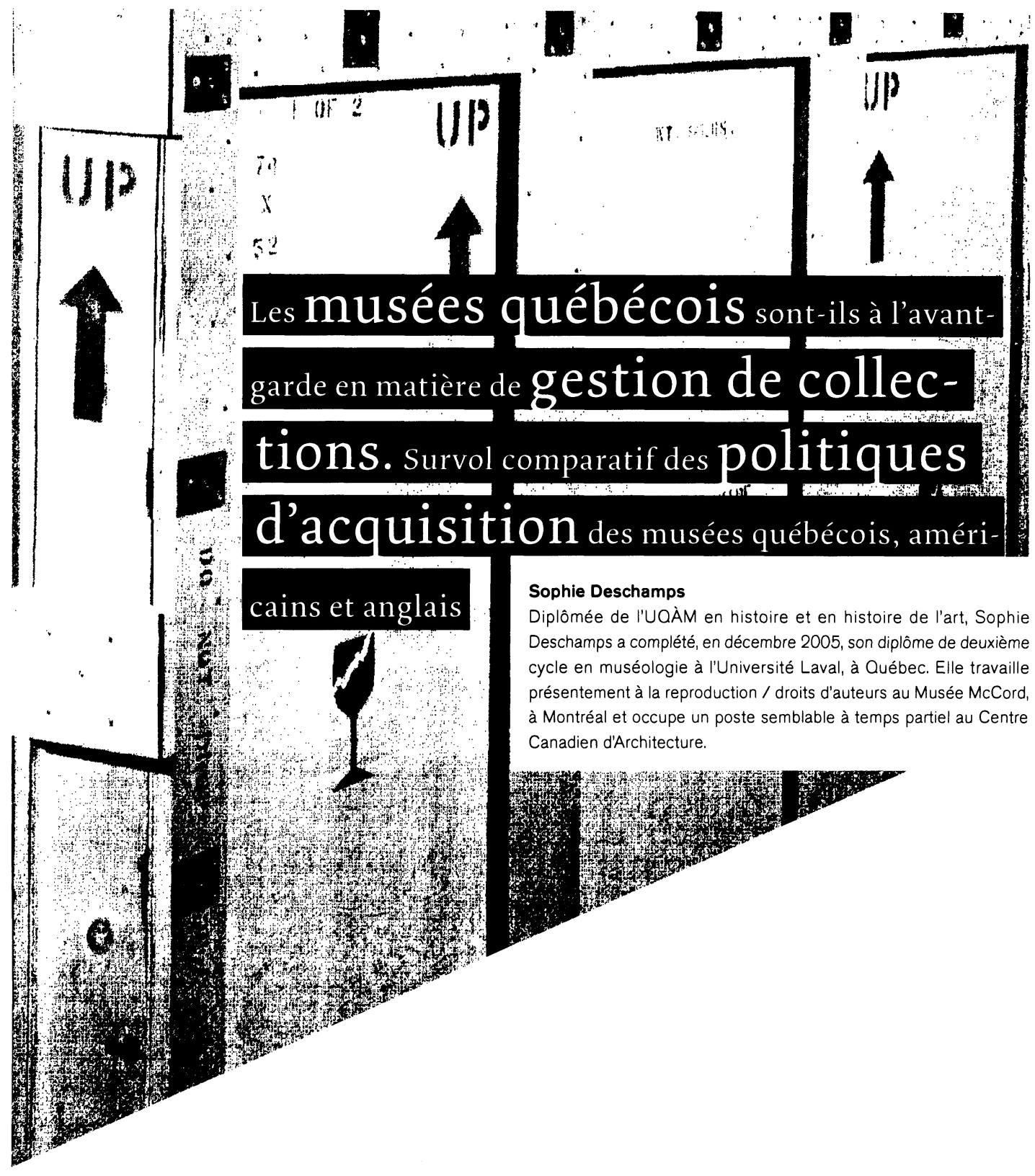


Cette analyse ${ }^{(1)}$ se veut un coup d'œil critique sur les politiques de collectionnement et, plus précisément, sur les politiques d'acquisition et leurs applications dans le monde muséal québécois. La gestion des collections doit être l'essence même de toute institution muséale. La conservation préventive, les démarches de restauration et la mise à jour des bases de données sont à l'origine du bon fonctionnement d'une institution, mais, pour maximiser son potentiel d'efficacité, celle-ci doit établir de solides politiques de gestion de ses collections et s'y plier en tout temps. L'analyse présentée ici est le fruit d'un travail qui s'échelonne sur huit mois. Plusieurs enquêtes ont été menées auprès de la communauté muséale québécoise et des recherches comparatives en Angleterre et aux États-Unis nous ont amenée à croire que la gestion des collections au Québec est déficiente et souffre de certaines carences en ce qui a trait aux politiques d'acquisition. Le Canada jouit, sans contredit, d'une grande avancée technologique en ce qui concerne la gestion des collections, que l'on parle de numérisation, d'expositions virtuelles ou de conservation préventive, mais les politiques qui régissent cette gestion demeurent trop flexibles ou sont carrément absentes des rapports annuels des institutions. Le problème se situe donc au centre de toute cette
Cette analyse est tirée de : DESCHAMPS, Sophie. Point d'étude : Les musées québécois en matière de gestion des collections. Analyse critique et survol comparatif des politiques d'acquisition et de gestion des collections des musées québécois, américains et anglais. Québec : essai déposé en décembre 2005, Université Laval, $127 \mathrm{p}$. 
pratique de gestion, à savoir que les fondements à l'origine des politiques d'acquisition sont inconséquents par rapport à la mission première du musée, car ceux-ci ne peuvent offrir des bases solides aux politiques qui tentent de s'ancrer dans des institutions constamment influencées par un contexte politico-culturel sans grande ambition de gestion. Tandis que certains gouvernements comptent parmi leurs objectifs culturels la conservation et l'acquisition des artefacts qui forment l'héritage national, la tendance canadienne est de s'intéresser davantage à l'ouverture aux publics par ailleurs de plus en plus nombreux et à la reconnaissance internationale.

Cette affirmation ne met pas en doute les compétences du ministère à s'occuper de la culture. Elle soutient simplement que la mission du musée est négligée par rapport aux ambitions d'unité nationale et de reconnaissance culturelle. Il y a plusieurs programmes d'aide aux musées qui sont une ressource importante de financement, mais la plupart de ces programmes sont axés sur les publics, les bâtiments ou les programmes éducatifs. D'un autre côté, il y a plusieurs organismes voués au patrimoine, comme le Réseau canadien d'information sur le patrimoine (RCIP), la Société des musées québécois (SMQ), le Musée virtuel du Canada (MVC) ou l'Association des musées canadiens (AMC), qui se penchent plus sérieusement sur la gestion des collections. Par l'analyse de leur mission et de leurs clauses relatives aux acquisitions, nous tenterons ici de définir l'approche canadienne de la gestion de collections.

Afin de positionner le contexte canadien, il est de mise de le comparer avec ce qui se fait ailleurs. Pour ce faire, nous avons brièvement étudié les contextes politico-culturels de l'Angleterre et des États-Unis. Cette analyse comparative permet d'établir la tendance canadienne de la gestion des collections par rapport à celle de ces deux pays. L'Angleterre et les États-Unis ont des modes de fonctionnement distincts. Ils représentent deux acteurs importants dans le développement des orientations canadiennes. Le mode de fonctionnement du milieu muséal canadien est fortement influencé par ce qui se fait en Angleterre, en raison de leur histoire commune, et par ce qui se fait aux États-Unis, qui, outre leur influence mondiale, sont les plus proches voisins du Canada. Lorsqu'une politique est mise de l'avant, certains objectifs précis en découlent. Ce sont ces objectifs qui sont analysés afin de déterminer l'idéologie nationale de ces pays en matière de gestion de collections et, plus précisément, de politiques d'acquisition.

Cette étude permettra de faire le point sur l'approche québécoise des politiques d'acquisition et de la gestion des collections et, espérons-le, sensibilisera le milieu muséal au problème à la base des écarts entre la mission et les actions du musée. 


\section{Deux discours, une seule orientation : le contexte politico-culturel du Québec}

Au Canada, la politique est formée de trois paliers de gouvernement : le fédéral, le provincial et le municipal. Le Québec se plie aux lois culturelles fédérales, mais crée aussi ses propres conventions. Même si le discours concernant la culture differe d'un palier de gouvernement à l'autre et les orientations sont les mêmes.

Le gouvernement du Canada définit une politique culturelle comme étant : «[...] la manifestation de la volonté du gouvernement d'adopter et de mettre en œuvre un ensemble cohérent de principes, d'objectifs et de moyens visant la protection et l'épanouissement de l'expression culturelle du pays " ${ }^{[2]}$. Dans ce contexte, "le rôle de l'État consiste à appuyer la démarche artistique, à assurer aux créateurs des conditions favorables à la pratique de leur art et à favoriser l'accès du grand public à leurs œuvres " " ${ }^{[3]}$. En réponse à cette définition, le ministère du Patrimoine canadien établit certains objectifs précis pour bâtir sa politique culturelle. Il s'agit d'objectifs fortement axés sur l'unité nationale, plus que sur la réelle préservation du patrimoine ou de l'héritage culturel. Ces objectifs comptent trois points : Protéger et affirmer la souveraineté canadienne, promouvoir l'unité nationale et promouvoir l'identité nationale canadienne. Le Canada est un pays multiculturel qui insiste et favorise grandement un discours entre ses communautés afin d'atteindre le but d'unité nationale qu'il s'est fixé.

Toutes ses actions et ses politiques en découlent. Les programmes culturels veulent appuyer la participation des différentes communautés à l'expression d'une culture nationale en facilitant l'accès à la vie culturelle afin de préserver l'héritage et le patrimoine canadiens. Dans son document Plan et priorité 2003-2004 ${ }^{(4)}$, le ministère du Patrimoine canadien exprime son souhait de remplir sa mission qui est de construire un Canada uni en promouvant la création artistique, la diffusion et la préservation des biens culturels canadiens, en établissant le lien entre les diverses communautés ainsi qu'en garantissant l'accès et en promouvant la participation des communautés à la vie culturelle. Par le biais de ces priorités, le but du gouvernement fédéral est de : "Connect Canadians across their difference, to link them to their history and to enable their diverse voices to participate in choosing the Canada they want " ${ }^{[5]}$.

Les politiques émanant du gouvernement fédéral seront donc fixées en regard de sa position sur l'unité nationale. Pour le ministère du Patrimoine canadien, les musées sont un excellent vecteur de diffusion du patrimoine. Ils préservent l'héritage culturel du pays, en plus de donner accès à la culture à de nombreux publics nationaux et internationaux. Plusieurs lois fédérales concernent donc ces institutions muséales, comme la Loi sur les musées, la Loi sur l'exportation et l'importation de biens culturels et la Loi sur le droit d'auteur qui « établit le droit économique et moral des créateurs de contrôler la

\section{[2]}

Parlement du Canada.

$\langle$ www.parl.gc.ca>

(consulté en décembre 2005).

[3]

Ibid.

[4]

Compendium of Cultural

Policies and Trends in Europe. <www.culturalpolicies.net>

(consulté en décembre 2005). [5]

Compendium of Cultural Policies and Trends in Europe. Plan et priorités 2003-2004. <www.culturalpolicies.net> (consulté en décembre 2005). 
Bibliothèque du Parlement Canada. Définition de la Loi sur le Droit d'auteur. < www.parl. gc.ca> (consulté décembre

2005)

[ 7 ]

Conseil des Arts du Canada. Rôle et mission du Conseil des Arts du Canada. <www. canadacouncil.ca> (consulté en décembre 2005) publication de leurs œuvres, d'être rémunérés et de protéger l'intégrité de leurs réalisations $"{ }^{[6]}$. Cette loi n'a pas de lien direct avec la gestion des collections au Canada, mais elle est importante en ce qui concerne les publications que font les musées et l'aliénation de certaines œuvres acquises par des musées et dont les artistes sont toujours vivants. La Loi sur l'exportation et l'importation de biens culturels, quant à elle, définit clairement ce qui peut être jugé comme bien culturel. De plus, une clause concernant le rapatriement des biens culturels nationaux favorise certaines acquisitions des musées d'État. Toujours en ce qui concerne les acquisitions, cette loi encourage les dons aux musées en accordant des reçus d'impôt aux donateurs. Le problème, alors, réside dans les politiques d'acquisition propres à chaque musée. Les musées acceptent souvent des dons qui ne correspondent pas à leur mission ou qui n'ont aucun intérêt de sauvegarde patrimoniale ou d'héritage national, en échange de reçus pour fins d'impôts. Ces artefacts ou ces œuvres occupent, dans les réserves, des places qui ne seront pas disponibles pour de futures acquisitions importantes. Cette question sera traitée plus loin dans cet article.

Outre les lois qui concernent les musées, le gouvernement a élaboré certains programmes d'aide financière afin de subvenir à leurs besoins. Ces programmes visent les projets concernant la production et la diffusion de la culture canadienne, l'ouverture aux publics, la reconnaissance nationale et internationale d'une institution, etc. En d'autres termes, ces programmes d'aide fédérale suivent le même axe de pensée que les politiques culturelles. La place de la gestion des collections et des politiques d'acquisition est restreinte par la Loi sur l'exportation et l'importation de biens culturels. Cependant, le Conseil des Arts du Canada, qui a comme mission "de favoriser et de promouvoir l'étude et la diffusion des arts, ainsi que la production d'œuvres d'art " ${ }^{[1]}$, offre un programme d'aide financière aux institutions pour l'acquisition d'œuvres originales de l'art contemporain canadien. Ce programme nommé Acquisition Assistance est offert aux galeries publiques d'art canadien ainsi qu'aux musées d'art qui démontrent un intérêt pour la diffusion et la préservation de l'art contemporain du Canada.

L'aspect "acquisition» de la mission du musée n'est donc pas négligé par le gouvernement, mais il est relégué à un rang inférieur, laissant le plus de place possible à la possibilité de reconnaissance internationale, d'unité nationale et d'ouverture aux publics.

Du côté provincial, le ministère de la Culture et des Communications s'est donné comme mission :

de favoriser au Québec l'affirmation, l'expression et la démocratisation de la culture ainsi que le développement des communications et de contribuer à leur rayonnement à l'étranger $[. .$.$] en suscitant le développe-$ ment régional, national et international des entreprises et des organismes engagés dans la culture et les communications. La mission du ministère 
place la population au cœur de ses préoccupations [et souhaite] favoriser l'accès des citoyens aux arts, à la culture et aux communications ${ }^{\left[{ }^{[8]}\right.}$.

Il s'agit d'un autre discours, moins nationaliste, mais tout aussi axé sur le développement et l'ouverture aux publics. Le Rapport annuel 2004-2005 fait état de cinq orientations ministérielles qui ont marqué les actions du provincial en matière de culture et de communications, pendant la dernière année. Ces orientations démontrent l'intérêt du ministère pour ce qui touche aux affaires culturelles :

- ORIENTATION 1 : Le soutien à la création, à la production, à la diffusion et à l'accès aux œuvres et aux contenus ;

- Orientation 2 : La participation active des citoyens et des citoyennes à la vie culturelle;

- Orientation 3 : Le développement des communications au profit de la vie démocratique;

- Orientation 4 : Le rayonnement et la défense des intérêts du Québec en matière de culture et de communication;

- Orientation 5 : L'adaptation du réseau d'action culturelle et de l'industrie des communications.

Diffusion; accès ; participation ; intérêts du Québec (provincial) / unité nationale (fédéral), tous ces mots traduisent une approche " désengagée " des gouvernements en ce qui concerne le problème de la gestion des collections au Québec. Il existe autant de politiques de collections que de types de musées. Étant toutes différentes, chaque institution muséale établit une politique qui lui est propre. Les musées sont conscients de leur mission au sein de la société, mais ils sont soumis à des conditions financières. Pour être admissibles aux programmes d'aide financière de l'État, les musées doivent rencontrer des critères d'admission qui découlent de la tendance gouvernementale en matière de culture. C'est pourquoi, bien que leur rôle soit aussi de privilégier le développement de leurs collections, les musées orientent leurs activités principalement en fonction des publics visiteurs.

Toujours pour appuyer ces propos, le Rapport annuel 2004-2005 du ministère de la Culture et des Communications établit les "priorités en matière de culture et de communication ". Outre l'amélioration des conditions socioéconomiques des artistes ou le soutien au cinéma et à la production audiovisuelle, les priorités reposent sur une reconnaissance du Québec à l'échelle internationale :

- La promotion de la proposition d'une convention internationale sur la diversité culturelle;

- La promotion de la culture québécoise à l'étranger.

La plupart des actions gouvernementales sont dirigées vers l'international. Avant d'explorer ces possibilités, il serait préférable de régler certains problèmes

\section{[8]}

Culture et communications

Québec. Mission.

<www.mcc.gouv.qc.ca>

(consulté en décembre 2005). 
fondamentaux au sein de la communauté muséale québécoise ou, du moins, il serait intéressant d'accorder un peu de place à ce qui se passe en coulisse dans les musées, car ces activités sont à la base de toute exposition, de tout programme d'interprétation et, enfin, ce sont ces activités qui, en bout de ligne, amènent le public à visiter l'exposition.

En octobre 2001, le gouvernement a signé un arrêté ministériel « concernant les normes en matière d'acquisition, d'utilisation et de gestion de droits des documents détenus par le gouvernement, les ministères et les organismes publics désignés par le gouvernement ", mais il ne concerne pas vraiment les institutions muséales. Cet arrêté prouve toutefois l'intérêt du gouvernement provincial pour certaines politiques d'acquisition concernant ses propres collections.

Le fait que chaque musée ait la charge de créer ses propres politiques de collectionnement permet peut-être aux gouvernements de se décharger des problèmes au détriment des institutions; mais, cela dit, tant que leur approche en ce qui concerne les politiques culturelles ne s'orientera pas davantage vers la conservation de l'héritage patrimonial, la gestion de ces biens et l'acquisition de ceux-ci, les musées continueront de considérer la gestion des collections comme étant de moindre urgence.

\section{Certains aspects du travail des organismes voués au patrimoine sont aussi un frein au développement des politiques de collectionnement}

L'analyse des politiques de trois organismes voués au patrimoine (RCIP, Réseau Info-Muse et Musée virtuel du Canada) démontre à quel point le Québec jouit d'une avancée technologique en ce qui concerne la gestion des collections. Malheureusement, l'approche et l'objectif de base menant à ces politiques de gestion de collections restent pour le moins superficiels. La vision d'ensemble des trois organismes se situe dans l'approche gouvernementale, c'est-à-dire une diffusion technologique des collections, facilitant l'accès au patrimoine culturel des publics nationaux et internationaux. Les technologies sont importantes pour la conservation préventive et elles permettent aux professionnels d'effectuer des recherches simplifiées dans les bases de données, mais est-il nécessaire d'en faire une priorité si les collections ne sont pas, au départ, régies par des politiques réfléchies et bien ancrées dans l'institution? Du point de vue de la technologie, de la numérisation, des logiciels de gestion, de la conservation préventive et de la diffusion internationale des artefacts sur Internet, le Canada joue un rôle de modèle. Il ne faut pas nier l'importance de ces nouvelles technologies, mais le musée devrait revenir à la condition que lui confere son statut de musée, qui est de se constituer une collection à l'aide de politiques claires axées sur sa mission. 
Un vaste organisme, le RCIP, a pour mission :

d'encourager le développement, la présentation et la préservation du contenu numérique patrimonial canadien. Cette mission met l'accent sur le désir de soutenir les musées au moment où ils s'engagent dans la voie dictée par l'importance actuelle de la gestion et de l'échange d'information sous forme électronique. [La vision du RCIP] est de brancher les publics canadiens et internationaux à notre patrimoine, en encourageant le développement, la présentation et la préservation du contenu numérique patrimonial canadien pour les générations présentes et futures ${ }^{[9]}$.

Dans le but d'atteindre ses objectifs, le RCIP s'appuie sur quatre priorités en lien avec l'approche gouvernementale de la culture :

1] LE PERFECTIONNEMENT DES COMPÉTENCES en améliorant la capacité des musées membres en profitant de l'émergence des technologies de pointe pour développer des services en ligne facilement accessibles, interactifs et personnalisés;

2] LA PROMOTION DE LACCÈS AU CONTENU PATRIMONIAL DANS LA VIE CIVILE ET SON UTILISATION en offrant un environnement virtuel didactique créé à partir de l'infothèque numérique des musées canadiens;

3] L'INTÉRÊT ET LA PARTICIPATION DU PUBLIC, grâce à l'analyse de la composition des audiences permettant de mieux cibler ses besoins et de mettre en œuvre des stratégies de communication efficaces;

4] LE RAYONNEMENT INTERNATIONAL en renforçant les collaborations permettant d'offrir toute la richesse du patrimoine canadien aux publics du monde entier ${ }^{[10]}$.

Le RCIP a mis sur pied des normes en gestion de collections qui vont de pair avec le Réseau Info-Muse, afin de guider les institutions muséales dans leur démarche d'informatisation des collections. Il s'agit d'outils documentaires qui facilitent la normalisation des données et des procédures d'enregistrement. Le RCIP est donc une source d'information très importante auprès de la communauté muséale et il constitue une ressource de base dans l'avancée technologique des musées. L'idée à l'origine de cette gestion de collections est similaire à l'approche des gouvernements, c'est-à-dire qu'elle met de l'avant l'importance de l'accès des publics aux collections numériques et la reconnaissance internationale des avancées technologiques. De plus, les acquisitions, qui sont la source même de toute numérisation de collections de musée, ne sont pas abordées dans les dossiers de normes du RCIP.

$\mathrm{Au}$ Québec, le Réseau Info-Muse est une base de données permettant l'échange d'information sur les collections des musées québécois. Relié au RCIP, cet outil a été mis en œuvre par la Société des musées québécois (SMQ) dans le
[9]

Réseau canadien d'information sur le patrimoine. Mission du RCIP. <www.rcip.gc.ca> (consulté en décembre 2005). [10]

Ibid. Objectifs et priorités du RCIP.<www.rcip.gc.ca> (consulté en décembre 2005) 
Société des musées québécois. Mission du Réseau Info-Muse. $<w w w . s m q . q c . c a>$ (consulté en décembre 2005).

[12].

Musée virtuel du Canada. Mission du MVC.

$<w w w$ museevirtuel.ca> (consulté en décembre 2005). but de favoriser l'automatisation des collections. La SMQ a aussi produit un guide papier sur la normalisation de l'enregistrement des collections dans la base de données afin que tous les utilisateurs du réseau utilisent les mêmes termes, les mêmes cotes et les mêmes normes d'enregistrement. Il s'agit donc d'informations de nature descriptive à des fins de normalisation de la gestion de collections. La mission du réseau est d'“assurer la présence d'un réseau d'échange de l'information sur le patrimoine québécois répondant aux besoins des institutions muséales et de la collectivité " [11]. Une fois de plus, ce réseau a été créé pour mettre en évidence l'avancée technologique du milieu muséal. En créant Info-Muse, la SMQ, tout comme le RCIP, démontre un intérêt marqué pour cette technologie de pointe et pour l'accès facilité et normalisé aux publics et aux chercheurs, mais néglige le fondement même de toute gestion de collections : des politiques d'acquisition réfléchies. Le réseau a été créé pour répondre aux besoins des institutions muséales, mais des renseignements concernant une normalisation des termes utilisés ne répondent qu'à un besoin parmi plusieurs autres qui sont tous aussi urgents.

De son côté, le Musée virtuel du Canada (MVC) est constitué d'un contenu multimédia novateur ayant pour mission "d'éveiller les publics de tous les âges à la diversité du patrimoine canadien en diffusant un service dynamique gratuit dans Internet, en français et en anglais " ${ }^{[12]}$. Ce site de musée virtuel a été lancé par le ministère du Patrimoine canadien en partenariat avec la communauté muséale canadienne afin d'aider la communauté patrimoniale à « tirer le meilleur parti possible des technologies de l'information. Une fois de plus, l'intérêt est porté sur l'accès international aux collections du patrimoine canadien, facilité par Internet, leader en matière de communication. Des expositions réunissant des collections de centaines de musées canadiens permettent aux publics internationaux d'accéder aux collections des grandes, mais aussi des petites institutions muséales. Les musées qui participent au MVC sont nommés "précurseurs " en la matière par le ministère du Patrimoine canadien, qui se fait une fierté d'une telle accessibilité de ses collections à l'étranger.

L'accès aux collections devient un événement assez superficiel si rien n'est tenté pour établir des politiques d'acquisition fermes et protéger la richesse culturelle du pays en conservant les collections qui la représentent. Une diffusion internationale de collections qui ne sont pas gérées adéquatement démontre un manque, non pas d'intérêt, mais d'une véritable compréhension des besoins fondamentaux de la gestion des collections au Québec.

Sans aucun doute, la gestion de collections souffre de carences, surtout en ce qui a trait aux politiques d'acquisition. Comment donc les institutions muséales peuvent-elles accorder l'énergie et le temps requis à la production de politiques réfléchies si le contexte économique, politique et culturel ne le permet pas ? L'importance accordée aux publics est nécessaire, mais à quel niveau ? Sans politiques d'acquisition fiables et à jour, sans politiques de 
gestion de collections réfléchies, les collections muséales ne pourront pas se développer dans un contexte adéquat. Si l'absence de politique d'acquisition ne permet pas un développement professionnel des collections, ce sont les expositions et, par le fait même, les publics qui en souffriront. Pourrait-on qualifier l'approche du gouvernement et des organismes voués au patrimoine comme trop objective, désengagée? Tout est mis en œuvre pour que les institutions canadiennes soient à l'avant-garde en ce qui concerne les nouvelles technologies, pour que le Canada soit considéré à l'étranger comme un leader en conservation préventive, en numérisation et en exposition virtuelle, mais les problèmes de fond ne sont pas abordés. Pour l'instant, il n'y a pas de crise majeure, mais, à force de reporter le problème et en raison du manque de politiques adéquates et réfléchies à la base de toute acquisition, les musées ne pourront plus garantir une des fonctions premières qui caractérisent leur mission. La conscientisation auprès des musées est effective, mais il est urgent que le gouvernement et les organismes voués au patrimoine passent à une approche davantage axée vers les réels besoins en ce qui concerne la gestion des collections, en gardant toujours à l'idée cette tendance d'ouverture aux publics qui assurera la stabilité du milieu muséal.

\section{Et si l'on regardait ailleurs!}

En Angleterre, le Department for Culture and Sport (DCMS), qui relève de l'autorité du Parlement, est responsable des politiques gouvernementales sur les arts, le sport, les bibliothèques, les musées et les galeries, les licences d'exportation de biens culturels, la gestion des collections d'art du gouvernement, etc. Son rapport annuel 2005, intitulé Making It Happen, fait état d'une revue majeure de tous les musées et des centres d'expositions d'Angleterre. Le document Understanding the Future (produit par le Department of Media, Culture and Sport Museums and Cultural Property) identifie les chemins à suivre pour les prochaines années afin de donner la possibilité aux musées de poursuivre leurs activités au sein de la communauté. Cette revue recueillera la vision, les conseils et les commentaires du milieu muséal afin d'établir ses besoins et de combler les lacunes pour les prochaines années. Understanding the Future marque l'intérêt du gouvernement à comprendre les musées afin d'envisager les solutions appropriées. Évidemment, l'intérêt des publics est aussi un enjeu important dans la création de politiques culturelles, mais cette ouverture aux visiteurs est traitée sur le même plan que les besoins fondamentaux des institutions muséales. 
Compendium of Cultural Policies and Trends in Europe. United Kingdom/Historical Perspective: Cultural Policies and Instruments. <www.culturalpolicies.net> (consulté en décembre 2005).
Pour le Department for Culture, Media and Sport, le but de chaque politique culturelle est :

- To make the best things in life available to the largest possible number of people;

- To increase access to and participation in the cultural and sporting life of the nation;

- To enhance the quality of the experience on offer, whetting people's appetite for excellence.

Avec ces objectifs, le ministère compte supporter et développer la qualité, l'innovation et la présentation d'un marché compétitif qui promeut le succès de l'Angleterre en ce qui concerne la culture. Aussi, il veut faciliter l'accès à une culture riche et variée en encourageant la formation et la préservation de collections formant l'héritage national. Toutes les politiques émanant du gouvernement anglais sont créées dans le but de donner l'occasion à tous les citoyens de développer leur talent afin d'atteindre l'excellence dans le domaine de la culture.

"Promoting social inclusion and neighbourhood renewal and its increasing commitment to investment in cultural capital (131), voila ce que propose le DCMS. Ces orientations ne sont pas contraires à celles que donne le gouvernement canadien à l'égard des politiques culturelles : accès aux publics, participation de la communauté... Les politiques culturelles anglaises restent en lien avec l'ouverture des musées aux visiteurs et la mission des musées, mais la différence entre les deux approches est que le DCMS cherche, grâce à ses projets, à combler les besoins fondamentaux des institutions muséales du pays. Il cherche à comprendre leur mode de fonctionnement et désire encourager la préservation des collections nationales.

Aussi, le conseil britannique des arts représente-t-il les autorités locales en matière de culture. C'est l'instance de décision à l'échelle régionale. Le conseil confere donc plus aisément au ministère la capacité de comprendre les problèmes des musées en région et d'entrevoir les solutions possibles afin de garder un bon rendement international. Ce besoin de reconnaissance pousse le gouvernement à exceller dans tous les domaines de la gestion des musées, y compris celui de la gestion des collections.

Le discours de l'Angleterre est comparable à celui du Canada, mais l'approche est plus impliquée en matière de gestion de collections. Il y a des programmes conçus pour mieux connaitre le milieu muséal et pour poser les actions appropriées en cas de crise potentielle. L'objectif de reconnaissance nationale présent dans chaque discours se définit par deux approches différentes dans chacun des pays, une davantage tournée vers l'avancée technologique et l'autre vers l'excellence de ses institutions. 
Aux États-Unis, le fonctionnement est très différent. Au Canada, il y a le ministère du Patrimoine canadien au fédéral et le ministère de la Culture et des Communications au provincial. En Angleterre, il y a un Department for Culture, Media and Sport, mais, aux États-Unis, aucun ministère fédéral n'est chargé de la culture, des arts ou des musées : «La plupart des musées sont indépendants de la puissance publique et font partie du secteur privé nonmarchand. [... ] Les musées américains ont donc toute latitude pour élaborer leurs propres normes professionnelles et ils se montrent traditionnellement très actifs et volontaires dans l'exercice de cette liberté. ${ }^{[14]}$ ) Le gouvernement n'a donc établi aucune approche en ce qui concerne la gestion des collections. Toutefois, quelques organismes voués au patrimoine sont très actifs et sont des leaders en la matière. Ceux-ci, en plus de mettre l'accent sur les nouvelles technologies, ont une approche réfléchie de ce que sont les problèmes de politiques d'acquisition. À ce titre, la situation de la communauté muséale américaine réunit l'approche "impliquée » de l'Angleterre et l'approche technologique « désengagée » du Canada.

En plus de certains organismes, tels que le Heritage Preservation et l'American Association of Museums (AAM), certains musées américains peuvent compter sur la Smithsonian Institution. La très grande association de musées qu'elle représente valorise les fonctions d'acquisition, de préservation, de recherche documentaire, d'interprétation et de diffusion des connaissances à un large public dans toute mission que se donne un musée. Pour elle, les collections sont au centre des activités que dirigent les institutions muséales. À cet effet, ces collections doivent être gérées adéquatement, de manière réfléchie, afin de se développer constamment, selon des critères établis dans la politique d'acquisition.

Les diverses approches en gestion de collections se ressemblent sur certains points. Elles estiment toutes que l'ouverture aux publics est un élément important du musée, elles considèrent toutes que les collections sont essentielles à cette ouverture des musées aux publics et elles aspirent toutes à une bonne gestion des collections, mais les actions et les faits sont dissemblables. Les gouvernements du Canada et du Québec misent sur l'avancée des technologies pour la conservation préventive et pour la diffusion des collections à un plus grand public. Ces deux gouvernements ont cependant une approche trop axée sur la reconnaissance internationale du pays et ils sont beaucoup moins interventionnistes et impliqués auprès des institutions muséales que ne l'est le Department for Culture, Media and Sport en Angleterre. Le DCMS mise davantage sur une bonne compréhension des besoins en gestion de collections afin d'offrir la meilleure base possible aux institutions qui prendront les mesures nécessaires à la création de politiques de gestion et d'acquisition réfléchies, comprises et appliquées rigoureusement par tous. Du côté américain, l'AAM et la Smithsonian Institution regroupent les deux approches (technologique

\section{[14]}

MALARO, Marie. " La gestion des collections et les cessions aux U.S.A $¥$. Les Nouvelles de I'ICOM, no 1, 2003.

<http://nic.icom.org> (consulté en décembre 2005). 
et impliquée). Ils se présentent comme des modèles significatifs de ce que devrait être la gestion de collections et leur approche réfléchie et complète permet la mise en œuvre d'une bonne politique d'acquisition.

Aucun modèle n'est parfait, mais une approche ouverte sur les publics qui permet en plus de réfléchir sur les besoins internes à la base de tout collectionnement semble être la voie idéale pour parvenir un certain équilibre du milieu.

\section{Que se passe-t-il au Québec?}

Vingt musées québécois ont participé à une cueillette d'information dans le contexte de notre étude qui s'est échelonnée sur huit mois. Trois tendances claires dans la pratique de la gestion des collections et des politiques d'acquisition en ressortent : l'approche minimaliste, l'approche classique et l'approche globale. L'idée première de cette critique n'était pas de classer les musées québécois selon les approches du Canada, de l'Angleterre ou des États-Unis, mais, à l'évidence, les musées québécois suivent un de ces trois modèles. Certaines institutions reconnaissent l'importance d'une bonne gestion des collections, mais n'en possèdent aucune réellement "réfléchie". Leurs idées s'appuient sur les objectifs gouvernementaux du Canada : leurs politiques sont donc axées sur l'ouverture aux publics et leurs acquisitions sont faites en fonction de l'intérêt de ces publics dans la majorité des cas étudiés. Aussi, plusieurs musées possèdent, à l'image des institutions d'Angleterre, une politique d'acquisition réfléchie, une politique de gestion de collections étoffée et cohérente avec la mission du musée et ils considèrent les intérêts des publics. Pour terminer, seulement trois institutions de notre échantillonnage ont une politique de gestion de collections réfléchie en plus d'une politique d'acquisition étoffée alliant l'importance des nouvelles technologies dans la conservation préventive, l'ouverture aux publics et la priorité accordée à la réflexion dans la création de politiques raisonnées, à l'image de ce qui se fait aux États-Unis.

Fortement axés sur les nouvelles technologies et l'ouverture aux publics : les musées à tendance "minimaliste»"

Les nouvelles technologies constituent pour les musées à tendance minimaliste un axe de développement très important des politiques de gestion des collections. Pour ces derniers, la conservation préventive est à la base même de toute gestion de collections - ce qui n'est pas incorrect -, mais elle est insuffisante, sans une réflexion plus poussée, pour acquérir la stabilité désirée. De plus, les organismes comme le RCIP, la SMQ et le Réseau Info-Muse, qui sont mentionnés dans plusieurs rapports annuels étudiés, privilégient certains des objectifs principaux des activités de gestion de collections, comme la 
numérisation active des collections et le téléversement de données dans Internet. Aussi, les expositions virtuelles sont-elles un des modes de diffusion de prédilection des musées de type minimaliste.

En ce qui a trait à leurs politiques d'acquisition comme telles, celles-ci s'appuient sur des critères relativement complets concernant les normes à respecter, qu'il s'agisse de la qualité de l'œuvre, de sa pertinence avec la mission du musée, de sa valeur monétaire et patrimoniale, de sa provenance bien documentée, de son état et, surtout, de son titre légal de propriété. En ce qui concerne les critères d'aliénation, tous les musées s'entendent pour dire qu'il devrait s'agir d'une " procédure claire et transparente quant à l'aliénation éventuelle de certaines œuvres de la collection " ${ }^{[15]}$.

Les musées de tendance minimaliste sont en majorité de petits musées, mais quelques grands musées adoptent aussi cette approche. Comme nous l'avions prévu, la majorité des petits musées établissent une tendance qui se rapproche très fortement de l'approche des gouvernements en matière de culture, car leur contexte économique et leur besoin de subvention l'exigent.

Fortement axés sur les politiques de gestion de collections étoffées et raisonnées et sur des politiques d'acquisition réfléchies : les musées de tendance classique

Bien que pour ces musées l'intérêt du public joue un rôle important dans le développement de l'institution, la bonne gestion de leurs collections est primordiale à leur évolution. Une bonne gestion des collections, pour les musées qui suivent cette deuxième tendance, signifie une réflexion menée à plusieurs niveaux afin de parvenir à une politique étoffée, définie et cohérente, qui procure des bases solides pour toutes politiques de gestion et d'acquisition stables.

Leurs critères d'acquisition sont plus sélectifs et rigoureux que ceux des musées minimalistes. En plus des normes de base, certaines clauses importantes sont détaillées, comme des champs de développement précis à respecter lors d'acquisitions, des analyses de la valeur muséographique des objets, des modes d'acquisition mieux définis ainsi qu'une clause concernant la révision de cette politique à des périodes régulières. L'aliénation, pour ces musées, consiste en une "mesure d'exception et de dernier recours " ${ }^{[16]}$ exécutée à la suite d'une révision ponctuelle des collections qui doivent évoluer avec le mandat du musée.

Ces musées sont donc très conscients des lacunes en ce domaine et tentent d'y remédier avec une approche très réfléchie et raisonnée en ce qui concerne leurs politiques de gestion et, surtout, leurs politiques d'acquisition. Par contre, ils ne s'impliquent pas du côté de l'avancée technologique. C'est-àdire que les musées « laissent le soin au RCIP de fournir le soutien technique,
[15]

Musée National des Beaux-Arts du Québec. Rapport annuel 2002-2003. p. 30.

[16]

Musée du Fjord. Politique de gestion des collections. Février 2002. p. 6. 
Musée canadien des Civilisations. Politique de gestion des collections. 1994. p. 3. la direction et l'orientation en ce qui a trait à l'informatisation des systèmes d'information sur les collections des musées " "11) et ne prennent pas vraiment d'initiatives dans ce domaine. Ils possèdent la base minimale en ce qui concerne la numérisation des collections, des bases de données et des expositions numériques (pour certains cas seulement), mais ils ne mettent pas l'accent sur ces projets.

Les musées ayant recours à l'approche globale

Ce dernier type d'approche est axé sur une politique de gestion de collections réfléchie et sur une politique d'acquisition étoffée, solidifiées par un raisonnement qui allie les nouvelles technologies et l'ouverture aux publics. Pour contrer le problème du surcollectionnement, il faut posséder une grande connaissance des collections conservées, il faut aussi disposer d'une bonne gestion de collections, stable et réfléchie, afin de maximiser le potentiel de l'institution. Les musées qui suivent cette approche l'ont bien compris. En mettant autant d'importance sur l'ouverture aux publics, sur la reconnaissance de l'institution dans le milieu muséal, sur les réflexions et le raisonnement à la base de toute bonne politique d'acquisition et en ne perdant jamais de vue l'objectif d'être à la fine pointe de la technologie sur les plans de la diffusion numérique et de la conservation préventive, certains musées ont atteint une stabilité sans pareil dans la gestion des collections au Québec. Leurs politiques d'acquisition sont complètes, basées sur des critères solides et détaillés et sur des conditions d'acquisition ou de refus bien définies, les rendant plus stables, rigoureuses et bien ancrées dans l'institution. Les lacunes observées par ces musées lors des examens annuels et des analyses de la gestion de leurs collections ont été partiellement comblées, car une institution qui prend conscience de ses problèmes et les comprend peut entrevoir des solutions efficaces. Les critères d'aliénation sont très rigoureux, mais il est spécifié, comme pour les autres institutions, que cette démarche (l'aliénation) ne doit être faite qu'en tout dernier recours.

L'approche globale devrait être envisagée par la majorité des musées québécois afin d'améliorer les conditions de gestion de collections et s'offrir des bases plus solides relatives aux politiques d'acquisition. Il est certain que les plus petits musées ne disposent pas d'assez de ressources pour modifier leur approche de la gestion des collections, ni pour s'imposer des critères rigoureux d'acquisition. Cela dit, notre étude a prouvé que certaines petites institutions y parviennent. En gardant les acquis des nouvelles technologies et en s'ouvrant sur la réflexion à la base de toute politique, les musées québécois augmenteront leur potentiel de gestion de collections, seront mieux informés sur leurs propres collections et seront aptes à établir une politique d'acquisition rigoureuse et ferme. Ainsi, le milieu muséal québécois atteindra la stabilité recherchée et règlera une partie des enjeux qui mettent en péril la survie des institutions au sein de la société québécoise. 
À la lumière de cette analyse, nous pouvons conclure qu'il y a bel et bien un problème en gestion de collections au Québec. En étudiant le contexte politico-culturel dans lequel évoluent les institutions muséales québécoises, nous comprenons un peu mieux les conditions qui prévalent à la création de ces politiques. La source du problème est double. D'un côté, les ministères n'accordent d'importance qu'au côté plus social du musée et établissent des programmes d'aide basés sur une approche désintéressée en ce qui concerne les fonctions de base des musées. D'un autre côté, les institutions muséales qui se retrouvent dans des positions économiques assez précaires doivent se soumettre à ces critères de sélection en matière d'aide financière et remplir les fonctions et les tâches que le gouvernement leur impose. Ces critères d'ouverture aux publics, de reconnaissance internationale et d'avancée technologique ne font pas partie des seules priorités des musées, mais l'octroi de subventions exige que les demandeurs y consacrent beaucoup de temps et d'énergie qu'ils devraient plutôt vouer aux problèmes de base dont souffre le milieu muséal. L'importance d'être à l'avant-garde en matière de technologie reste un champ remarquable au Québec et les ministères l'ont bien compris. De plus, plusieurs organismes influents s'y consacrent avec énergie, comme c'est le cas du RCIP ou du Réseau Info-Muse.

Le milieu muséal manque sans doute d'information concernant les politiques solides de gestion de collections et les politiques d'acquisition rigoureuses à adopter. Comparativement à ce qui se fait en Angleterre, cette fonction du musée a été beaucoup moins exploitée, moins mise de l'avant et probablement moins bien comprise au Québec. On remarque, depuis une dizaine d'années, une prise de conscience des problèmes qu'amène une mauvaise gestion de collections, mais les étapes pour combler les carences demeurent nombreuses. Il ne faut pas perdre de vue l'importance des technologies de pointe dans le développement d'une diffusion à grande échelle et aussi l'avantage qu'elles apportent à la conservation préventive. Les musées québécois doivent absolument réfléchir à la question de la gestion de collections et des politiques d'acquisition en revenant aux fonctions premières du musée, en gardant les acquis technologiques du XXI ${ }^{e}$ siècle.

Plusieurs recommandations en vue de l'uniformisation des politiques muséales au Québec découlent de ce constat. En voici quelques-unes :

1] Comme le recommandent aussi le Conseil international des musées et l'Association des musées canadiens et afin d'aider la communauté muséale québécoise à faire le point sur les avancées en matière de politique de gestion de collections et en ce qui concerne les acquisitions, les musées québécois devraient diffuser leurs politiques de gestion de collections et leurs politiques d'acquisition dans toute la communauté muséale québécoise. 
1] Toutes les politiques de gestion de collections et les politiques d'acquisition devraient être pensées et réfléchies en regard de la mission et du mandat de chaque institution muséale.

2] Afin de profiter d'une meilleure gestion des collections et pour éviter le surcollectionnement, les institutions muséales devraient instaurer une clause dans leur politique de gestion de collections concernant la révision des collections et des politiques d'acquisition au moins aux trois ans.

La publication et la diffusion, dans le milieu muséal, des politiques d'acquisition et de gestion de collections déjà établies dans certains musées jouent un rôle d'une importance capitale. Premièrement, cette diffusion permettra au milieu de faire le point sur les connaissances dans le domaine au pays; deuxièmement, ces exemples de politiques amèneront certainement une prise de conscience collective sur l'importance de concevoir des bases solides sur lesquelles se développera l'institution. En s'attardant plus amplement sur le sujet, les musées apprendront à se connaître davantage et à connaître leurs collections. Ils seront ainsi aptes à entrevoir des solutions efficaces.

\section{Summary}

Are Quebec museums at the avant-garde of collections management? A comparative survey of Québécois, American and English

collections management and acquisitions policies.

Collections management policies and acquisitions policies in particular are not part of the main activities of Quebec's museums. Yet, if conceived thoughtfully they allow museums to endow themselves with a solid basis to better develop within society. It is essential that museums become aware that a lack of proper collection policies will bring them deficiencies in collection management.

This article proposes some solutions to address the deficiencies in collections management and reflections for a more in-depth study of issues in collections management and acquisitions policy in Quebec's museum realm. It is the result of an in-depth analysis of facts regarding the internal functioning of Quebec's museums. It intends to make its point by establishing a connection between the politico-cultural context in which museums evolve and the secondary position that collections policies are given in many institutions. By studying and comparing the English practice, (our historic model) and that of the United States (our close neighbour and important 
influence) it will be possible to bring out three major Quebec tendencies as to the use and implementation of collections and acquisitions policies. A survey of twenty museums across Quebec and Canada was conducted in order to understand the facts and target the actual needs in this area. The annual reports of many of Quebec's establishments, as well as the acquisitions policies and the broad guidelines concerning the development of collections were analyzed. Many museums have a coherent and substantial collections policy, as well as a well thought-out acquisitions policy, but do not grant new technologies the value required. Some museums recognize the importance of endowing themselves with sensible collections management policies but only follow minimal acquisitions policies, whereas others adopt substantial and considered collections policies, fully defined acquisitions policies supported by new technologies that allow more accessibility to their publics.

Without a doubt, collections management in Quebec suffers from deficiencies, especially as concerns acquisitions policies. Governments and heritage organizations do their best to insure that Canadian institutions are at the forefront of new technologies, so that Canada can be considered an international leader in preventative conservation, digitization, and virtual exhibition. However, basic problems are not being dealt with. At the moment, there is no major crisis but museums will no longer be able to guarantee the effectiveness of one of the primary functions of their mission (that is collecting) if they don't implement adequate and scrupulous policies for all acquisitions. The diverse approaches studied in collections management resemble each other on various points. The Canadian and Quebec governments privilege an approach that tends towards international recognition of their museum practices rather then getting involved and intervene in these institutions. With the use of new technologies and a due consideration of their policies, Quebec's museums will increase their collections management potential, will be more informed about their own collections, and will be apt to establish a rigorous and firm acquisitions policy. Thus, Quebec's museums will attain the stability they seek and address some of the issues that jeopardize the survival of these institutions at the heart of Quebec society. 